\title{
ROSAT AND OPTICAL OBSERVATIONS OF SUPERSOFT X-RAY SOURCES
}

\author{
K. REINSCH ${ }^{1}$, A. VAN TEESELING ${ }^{1}$, K. BEUERMANN ${ }^{1,2}$, \\ H.-C. THOMAS ${ }^{3}$, T. M. C. ABBOTT ${ }^{4}$ \\ 1. Universitäts-Sternwarte, Geismarlandstr. 11, \\ D-37083 Göttingen, Germany (reinsch@usw050.Inet.gwdg.de) \\ 2. MPI für Extraterrestrische Physik, Garching, Germany \\ 3. MPI für Astrophysik, Garching, Germany \\ 4. ESO Chile, current address: CFHT, Hawaii, USA
}

\begin{abstract}
We present X-ray, UV and optical observations of the Galactic supersoft X-ray source (SSS) RX J0019.8+2156 and first results of our study of the X-ray and optical long-term variability of bright SSS.
\end{abstract}

\section{Introduction}

Luminous supersoft X-ray sources (SSS) have been established as a new and distinct class of objects by the discovery of some 20 objects during the ROSAT mission, which show properties similar to the prototype system CAL 83 (for a recent review see Hasinger 1994). Several models, including accretion onto black holes and neutron stars accreting above the Eddington rate, have been proposed to explain the nature of SSS. The most prominent interpretation considers a white dwarf which accretes matter from its more massive main sequence secondary at a rate just sufficient to permit (quasi-) stable nuclear burning near its surface (van den Heuvel et al. 1992).

\section{The bright Galactic source RX J0019.8+2156}

Our complete sample of bright, soft, high-galactic-latitude ROSAT sources contains just one Galactic object which bears strong similarity to the LMC SSS: RX J0019.8+2156 (Beuermann et al. 1995). The system displays quasisinusoidal modulations of the optical, UV and X-ray light curves, as well as regular radial-velocity variations with a $15.85 \mathrm{~h}$ period which we interpret as the binary orbital period. Time-resolved spectroscopy of RX J0019+21

423

A. Evans and J. H. Wood (eds.), Cataclysmic Variables and Related Objects, 423-424.

(C) 1996 Kluwer Academic Publishers. Printed in the Netherlands. 
shows a complex structure and orbital variability of the Balmer and HeII line profiles, and a P Cygni absorption component in the Balmer lines.

$\mathrm{X}$-ray luminosity and temperature seem to be lower in RX J0019+21 than in CAL 83, RX J0513-69 and RX J0925-47. The simplest explanation is that the hot component in RX J0019+21 is a white dwarf with an accretion rate of $\sim 10^{-7} \mathrm{M}_{\odot} \mathrm{yr}^{-1}$, which just fails to allow stationary hydrogen burning. The system therefore may experience weak shell flashes, being currently in a luminosity minimum. This is supported by the optical long-term behaviour of RX J0019+21, which shows high and low states lasting about 20 yr each (Greiner \& Wenzel 1995).

\section{Long-term variability of supersoft sources}

Besides orbital variability, most of the SSS are highly variable on different time-scales down to weeks (or even less). We have studied the X-ray and optical variability of several bright SSS to obtain constraints for different models and to assess the evolutionary status of individual systems.

While the soft X-ray flux of RX J0019+21 has been fairly constant on time-scales of years since the ROSAT All-Sky Survey (RASS) in 1990, the $0.1 \ldots 2.4 \mathrm{keV}$ flux of the LMC source RX J0549.9-7151 has decreased by more than a factor of 200 since the RASS observation and a pointed PSPC observation of CAL 87 containing RX J0549-71 as a serendipitous source (Cowley et al. 1993). The X-ray flux of RX J0439.8-6809 (Greiner, Hasinger $\&$ Thomas 1994) is variable by a factor of 2 on time-scales as short as a few seconds. On longer time-scales, however, the source has been remarkably constant since the RASS. The variability of the suspected faint optical counterpart is constrained to be less than $\sim 0.5 \mathrm{mag}$.

\section{References}

Beuermann, K., Reinsch K., Barwig, H., et al., 1995, A\&A, 294, L1

Cowley, A.P., Schmidtke, P.C., Hutchings, J.B., et al., 1993, Ap. J., 418, L63

Greiner, J., Hasinger, G., Thomas, H.-C., 1994, A\&A, 281, L61

Greiner, J., Wenzel, W., 1995, A\&A, 294, L5

Hasinger, G., 1994, Reviews in Modern Astron., 7, 129

van den Heuvel, E.P.J., Bhattacharya, D., Nomoto, K., Rappaport, S.A., 1992, A\&A, 262, 97 